See discussions, stats, and author profiles for this publication at: https://www.researchgate.net/publication/322514787

\title{
A review of aligners for protein protein interaction networks
}

Conference Paper · May 2017

DOI: 10.1109/RTEICT.2017.8256879

CITATIONS

READS

128

4 authors, including:

Anooja Ali

Reva University

11 PUblications 5 CitATIONS

SEE PROFILE

Dr.Siddanagouda Somanagouda Patil

-1) University of Agricultural Sciences, Bangalore

27 PUBLICATIONS 58 CITATIONS

SEE PROFILE

Some of the authors of this publication are also working on these related projects:

Project NISAGENET project View project

Project cervical cancer_Bioinformatics View project

R. Vishwanath

Reva University

8 PUBLICATIONS 4 CITATIONS

SEE PROFILE 


\title{
A Review of Aligners for Protein Protein Interaction Networks
}

\author{
Anooja Ali, REVA University, Bangalore, anoojaali@gmail.com, \\ Viswanath R. Hulipalled, REVA University, Bangalore, viswa.gld@gmail.com, \\ S. S. Patil, UAS, Bangalore, spatiluasb@gmail.com, and \\ Venugopal K. R., UVCE, Bangalore, venugopalkr@gmail.com
}

\begin{abstract}
Protein Protein Interaction (PPI) can be considered as network. Alignment is the process of mapping nodes from one network to another network. The main objective of network alignment is to identify small, well defined interactome units such as protein complexes or conserved pathways that are analogous in the input network. Network alignment uncovers the relationship between protein complexes and functions. Similarity between two graph structures can be identified by evaluating the topology. Network alignment identifies either topological or sequential similarity. Gene annotations reveal the functional or sequential similarity and it can be evaluated based on semantic similarity. In this paper, we review the various network aligners and classify them according to the methodologies. We discuss the different evaluation metrics and the popular databases of protein interactions.
\end{abstract}

Keywords - gene annotations, network alignment, protein protein interaction, sequential similarity, topological similarity

\section{INTRODUCTION}

Proteins are the macromolecules responsible for majority of activities in any living organism. Protein interactions can be visualized as a network, where proteins represent vertices and their interactions denote edges [1]. Representing biological data as graph or network speeds up the analysis and two networks can be compared by graph alignment [2]. Network alignment is the equivalent to sub graph isomorphism and it is NP complete. PPI interactions can be transient or stable.

Network alignment can be global network alignment or local network alignment. Local network alignment matches smaller regions of similarity across network. Local network alignment considers substructure mapping and global alignment considers the entire network and starts mapping [3]. Network alignment identifies either topological or sequential similarity [4]. Topological similarity is also referred as Neighborhood similarity or Signature similarity. Sequential similarity can be biological similarity or functional similarity. Nodes present in the PPI network will be associated with annotation terms or Gene ontology [5]. Several wide advances have been proposed for network alignment in past few years, most of the approaches are derived from the previous ones and few of them are widely different. The parameters that were used to evaluate solutions are varying over the years.

In this paper, we survey the different topological and biological assessment for PPI network and the evaluation of various algorithms used for network alignment. The rest of the paper is organized as follows: The next section introduces the problem of pairwise network alignment. Then, we evaluate the different measures suggested for topological and biological assessment. Following that, an assessments of popular network aligners are summarized. Finally, the datasets used for alignment, scope of future work and concluding remarks are presented.

\section{NETWORK ALIGNMENT}

This section interprets the meaning of pairwise network alignment, the different assessment strategies and how it can be extended to multiple network alignment.

Consider two different networks $\mathrm{N} 1$ and $\mathrm{N} 2$, where $\mathrm{N} 1=$ $(\mathrm{V} 1, \mathrm{E} 1)$ and $\mathrm{N} 2=(\mathrm{V} 2, \mathrm{E} 2)$. The individual edge $(\mathrm{u}, \mathrm{v})$ highlights the interaction between proteins. The aim of network alignment is to calculate the injective function $f$ as mentioned in (1).

$$
f(\mathrm{u})=\{\mathrm{v} \text {, where } \mathrm{u} \in \mathrm{N} 1 \text { and } \mathrm{v} \in \mathrm{N} 2\}
$$

If all the nodes are aligned, then the alignment is completely defined or else it is partially defined. In the process of aligning it may be possible to find more than one alignments in the second network, so it advised to find the best alignment using scoring functions [2].

\section{A. Topological Assessment}

Topology of the network is assessed by several metrics. Edge Correctness (EC) is a standard metric. The percentage of edges aligned denotes the effectiveness of EC [3]. EC aligns a sparse network to a dense network and due to these multiple alignments may be generated for an edge and Induced Conserved Structure (ICS) penalizes this [6]. ICS aligns dense to sparse alignment. If two proteins have momentous number of common neighbor, they will be meticulously related. Combining the prediction from several topological methods and merging similar predictions based on overlap score identifies the excelling prediction.

\section{B. Semantic Similarity Assessment}

Semantic similarity measures can be either annotation based or ontology based approaches [7]. In graph structure a graph is constructed with ontology terms as nodes and edges as relationship between terms. Annotations are terms that focus on the functionality of genes or proteins. Ontology measures or structure based methods are broadly divided into edge based and node based methods [8]. Semantic similarity has the peak predictive score in measuring true PPI interactions. 


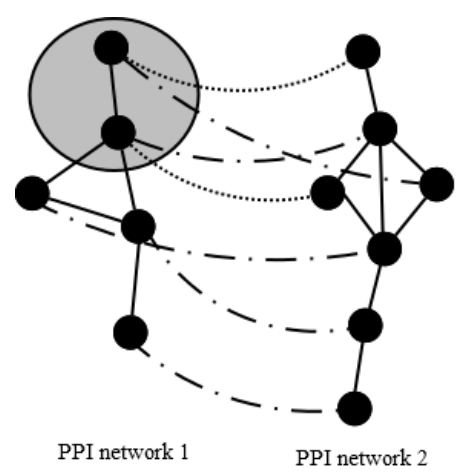

a) Local Netwok Alignment

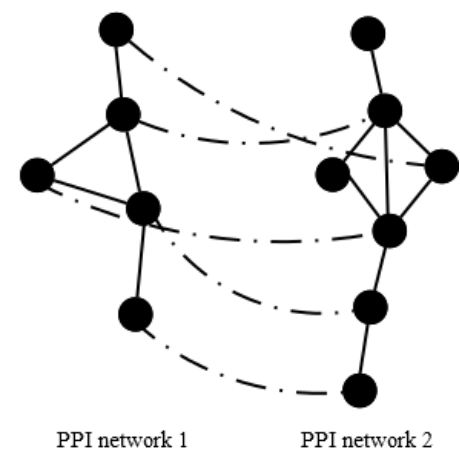

b) Global Network Alignment

Figure 1. Local network alignment versus Global network alignment. (a) Nodes in the shaded region can have two different alignments differentiated by separate dashed lines. (b) Global network alignment aligns the two networks probing a common sub network.

Semantic similarity measures can be group wise or pairwise. In pairwise the similarity between annotations of terms are evaluated as represented in (2). If the annotations of node ni and node nj are represented as ann(ni) and ann(nj) respectively, then functional similarity is evaluated as

$$
\operatorname{Fsim}(n i, n j)=\frac{\operatorname{ann}(n i) \operatorname{ann}(n \bar{p})}{\operatorname{ann}(n i) \cup \operatorname{ann}(n j)} .
$$

Group wise approaches are related to ancestor graphs. Resnik's [9], Lin's [10] and Jiang's [11] approaches examines the pairwise similarity. Semantic similarity can be measured at any level of orthogonal ontology [12]. The threshold value of similarity defines the minimum amount of similarity required for alignment. PPI network can be subdivided into modules based on functionality or biological similarity [13]. Many researchers have proposed methods to scale large PPI network into smaller graphs. If the topological and functional similarities are same, then they can be merged under a single functional module [14].

\section{GLOBAL NETWORK ALIGNMENT}

Pairwise network alignment forms the base for Local network alignment and they fall of due to inconsistency [15]. Fig. 1 shows the comparison between local network alignment and global network alignment. The main intention of Global aligners is to find a mapping node or to mark the node as gap node. Alignments within a cluster can be even done by inserting gap at different locations [16]. Global aligners are classified into IsoRank aligners and GRAAL based aligners.

\section{A. IsoRank Aligners}

IsoRank aligners are based on the compatibility between sequence order and topological structure [17]. Similarity between proteins is estimated using a version of Google's PageRank algorithm [18]. Nodes are aligned from one network to another if their neighbors are similar. IsoRank creates a functional orthologous set or a cluster of orthologous genes
[19]. IsoRank uses greedy pairwise alignment with sequence based BLAST scores.

$$
\mathrm{R}=\alpha \mathrm{AR}+(1-\alpha) \mathrm{E}
$$

In (3), $\mathrm{R}$ denotes the mapping, A denotes the Eigen vector. $\mathrm{E}$ is the normalized sequence similarity. Value of $\alpha$ can be 0,1 or any value between them. If $\alpha=1$, only network information is considered and if $\alpha=0$, only sequence information is considered. Any value in between them considers both topological and sequence similarity.

Increasing the number of true positives can increase the performance of IsoRank but the possibility of over fitting must be taken care. Network similarity decomposition (NSD) improves IsoRank by increasing the speed of node similarity calculation and preprocessing each input graph separately [20]. The drawback of NSD is that it cannot run on higher networks.

Upgraded version of IsoRank is IsoRank Nibble (IsoRankN) relies on rankings that are specific to node [21]. It uses iterative spectral clustering algorithm and generates the final alignment. IsoRankN outperform IsoRank and Græmlin 2.0 [22] in coverage and consistency. IsoRankN generates many to many mapping and the evaluation of all these mapping is not feasible because the size of iterative spectral matrix is large [23].

\section{B. GRAAL Aligners}

Graph Alignment Aligners (GRAAL) evaluate graphlet degree signature. Proteins often change their interaction to perform multiple biological functionalities and so alignments based on topological similarities are sounder than considering solely sequential similarity. GRAAL aligners are mainly GRAAL [24], H-GRAAL [25], MI-GRAAL [26], C-GRAAL [27] and L-GRAAL [28].

GRAAL is the premier algorithm that considers only topological similarity. It identifies a seed vertex and from this this seed vertex it aligns vertices greedily on radius measurement. 

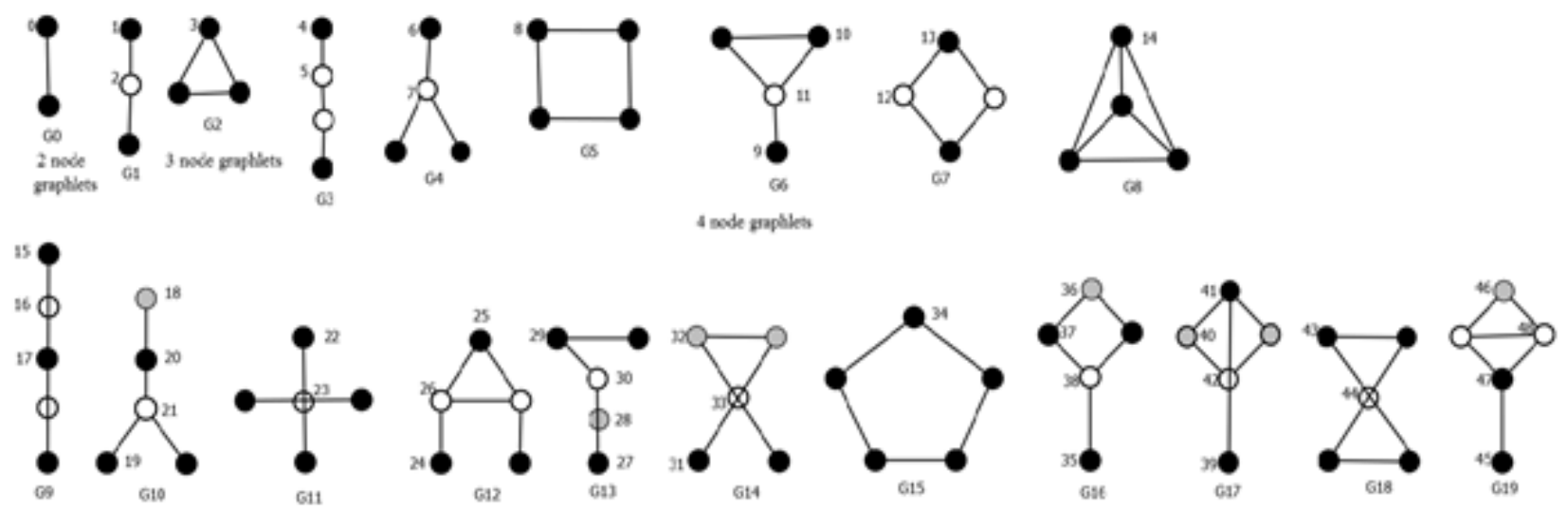

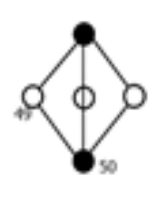

$\cos$

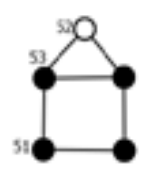

ต2:

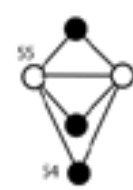

$6 n$

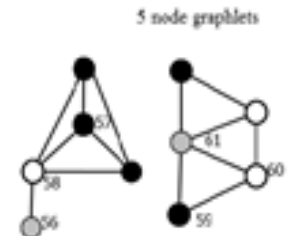

ars

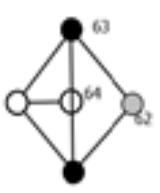

a.2

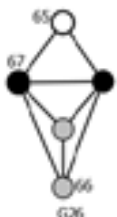

nole srablikts

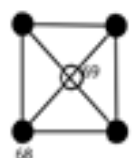

20

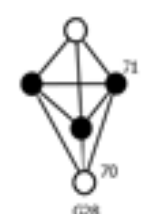

$\omega$

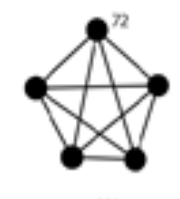

629

Figure 2. Graphlets ranging from 2 nodes to 5 nodes represented from G0 to G29.The 73 different orbits are labelled. This figure is adopted from [24].

Graphlet is an induced sub graphs and graphlet degree is the number of graphlet that is touched by each node. Nodes present in a graphlet can vary from a minimum of two nodes to a maximum of five nodes, as shown in Fig. 2. Automorphism orbit of graphlet infer the 73 different interactions or edges between a node and graphlet.

Hungarian GRAAL (H-GRAAL) follows minimum weight bipartite method like IsoRank. Hungarian algorithm ensures that no two nodes in first network is aligned to the same node in second network [29]. Matching based Integrative GRAAL (MI-GRAAL) creates a confidence matrix by integrating several biological and topological similarity metrics. Common Neighbors based GRAAL (C-GRAAL) explores nodes neighborhood similarity. Lagrangian Graphlet based Aligner (L-GRAAL) considers graphlet based interaction with Legrangian relaxation with graphlet of size two to four. Five node graphlet is not considered. It considers function protein conservation and biological protein conservation.

\section{PPI DATASETS}

The NP hard nature of the network alignment makes it difficult. The lack any perfectly guaranteed aligner complicates the alignment. IsoBase [30] and NAPAbench [31] are the popular datasets used for network alignment.

The other databases include Human Protein Reference Database (HPRD) [32], BioGRID [33], Database of Interacting Proteins (DIP) [34] and Munich Information center for Protein Sequences (MIPS) [35]. Table 1 indicates the number of edges and nodes present in the PPI network for different species like yeast, fly, human and mouse. It is obtained from IsoBase web interface.

978-1-5090-3774-9/17/\$31.00 @ 2017 IEEE
IsoBase is a real-time dataset for global network alignment. It facilitates browsing, searching and querying of a dataset. It is obtained by combining several other datasets. NAPAbench is a synthetic dataset, proposed in 2012. Synthetic datasets play a major role in validation of network alignments. NAPAbench dataset can be denser or sparser than real time PPI network for different suites. Synthetic dataset can mimic any real datasets.

\section{OPEN RESEARCH PROBLEMS}

Analysis of literature survey indicates there are a few avenues for future work. By considering those in mind, we list out a few open research problems on network alignment.

\section{A. Weightage Analysis of Similarity Feature}

It is difficult to identify the weightage or the priority of a similarity feature when several similarity features are to be considered. Alignment begins the highest scoring pair and it extends greedily from the seed pair. The challenge in developing an aligner is the similarity evaluation in terms of topological and biological similarities. Topological similarity considers maximum weight bipartite matching problem. Existing aligners generate heuristic algorithm to develop alignment. When proteins change their location their functionality also changes.

TABLE 1. THE NUMBER OF NODES AND EDGES OF DIFFERENT SPECIES FROM ISOBASE DATASET.

\begin{tabular}{|l|l|l|}
\hline Species & No: of Nodes & No: of Edges \\
\hline $\begin{array}{l}\text { Saccharomyces cerevisiae } \\
\text { (Yeast) }\end{array}$ & 6,659 & 38,109 \\
\hline Drosophila melanogaster (Fly) & 14,098 & 26,726 \\
\hline Homo sapiens (Human) & 22,369 & 43,757 \\
\hline $\begin{array}{l}\text { Mus musculus } \\
\text { (Mouse) }\end{array}$ & 24,855 & 452 \\
\hline
\end{tabular}


IEEE International Conference On Recent Trends In Electronics Information Communication Technology, May 19-20, 2017, India

TABLE 2. SUMMARY OF DIFFERENT ALIGNERS

\begin{tabular}{|c|c|c|c|}
\hline Aligner & Year & Methodology & Drawback \\
\hline IsoRank & 2007 & $\begin{array}{l}\text { Combines Sequential } \\
\text { and Topological } \\
\text { similarity, Proteins are } \\
\text { matched if neighbors } \\
\text { can be matched. }\end{array}$ & $\begin{array}{l}\text { True positives } \\
\text { increase over } \\
\text { fitting. }\end{array}$ \\
\hline IsoRankN & 2009 & $\begin{array}{l}\text { Iterative Spectral } \\
\text { Clustering Algorithm, } \\
\text { Proteins are matched if } \\
\text { neighbors can be } \\
\text { matched. }\end{array}$ & $\begin{array}{l}\text { Many to many } \\
\text { mapping and } \\
\text { Iterative nature of } \\
\text { spectral matrix }\end{array}$ \\
\hline GRAAL & 2010 & $\begin{array}{l}\text { Graphlet Degree } \\
\text { Signature, consider only } \\
\text { topological similarity. }\end{array}$ & $\begin{array}{l}\text { Loses power in } \\
\text { discriminating } \\
\text { between vertex } \\
\text { pairings. }\end{array}$ \\
\hline H-GRAAL & 2010 & $\begin{array}{l}\text { Hungarian Algorithm } \\
\text { with Graphlet Degree } \\
\text { Signature, consider only } \\
\text { topological similarity. }\end{array}$ & Higher Runtime. \\
\hline $\begin{array}{l}\text { MI- } \\
\text { GRAAL }\end{array}$ & 2011 & $\begin{array}{l}\text { Several topologies } \\
\text { matching methods are } \\
\text { integrated together. }\end{array}$ & $\begin{array}{l}\text { Higher time for } \\
\text { integrating } \\
\text { different metrics. }\end{array}$ \\
\hline NSD & 2012 & $\begin{array}{l}\text { Proteins are matched if } \\
\text { neighbors can be } \\
\text { matched. }\end{array}$ & $\begin{array}{l}\text { Cannot run on } \\
\text { higher networks. }\end{array}$ \\
\hline C-GRAAL & 2012 & $\begin{array}{l}\text { Nodes are matched if } \\
\text { they have common } \\
\text { neighbor and consider } \\
\text { only biological } \\
\text { similarity. }\end{array}$ & $\begin{array}{l}\text { Poor vertex } \\
\text { alignment } \\
\text { performance. }\end{array}$ \\
\hline L-GRAAL & 2015 & $\begin{array}{l}\text { Legrangian Relaxation } \\
\text { with Graphlet degree } \\
\text { signature. }\end{array}$ & $\begin{array}{l}\text { Graphlet size is } \\
\text { limited to } 4 \text {. }\end{array}$ \\
\hline
\end{tabular}

\section{B. Parametization for the Alignment of different type of Networks}

Identification of the correct parameterization for the alignment of different type of networks is a challenging problem. An advance knowledge about the species or their phylogenic information gives better performance. Aligners like Græmlin consider knowledge about PPI network is insufficient for alignment. So, it requires phylogenic information also for alignment. Thus it is not advised to compare the performance of these aligners with any other. Improvements on the topological metrics are progressing. Higher value of topological matrix comes at the expense of biological measurements. Table 2 gives a summary of various aligners surveyed. It compares the methodologies of different aligners and highlights the drawback of those aligners.

\section{Larger Datasets}

Network alignment can be extended to any larger datasets. Alignment of larger biological networks is an unexplored area of research. Greedy aligners could be extended to any pairwise alignment of larger datasets.

\section{CONCLUSION}

In this paper, we survey the different aligners for protein network alignment. First, we define the problem of pairwise network alignment. Following that, we evaluate the different metrics for topological and biological assessment. Later an assessment of the major state of art network aligners are explained with their corresponding methodologies. Finally, the real and synthetic datasets used for alignment, scope of future work and concluding remarks are presented.

Computation of similarity between nodes in terms of topological and functional similarity with different metric is also improving. Enhancing the performance of existing aligners by improving the time complexity and evaluation metric can improve PPI network alignment. The performance of aligners also changes according to the dataset used. Constructing a framework for evaluating aligners with common evaluation metric and same dataset will help biologist to decide the best aligner to use.

\section{REFERENCES}

[1] A. Elmsallati, A. Msalati, and J. Kalita, "Index Based Network Aligner of Protein-Protein Interaction Networks," IEEE/ACM Transactions on Computational Biology and Bioinformatics, vol. PP, no.99, pp.1-1, in press.

[2] Connor Clark, Jugal Kalita, and Ahed Elmsallati, "Global Alignment for Protein Protein Interaction Networks: A survey," IEEE/ACM Transactions on Computational Biology and Bioinformatics, vol.13, No.4, pp-689-705, July/August 2016.

[3] O. Kuchaiev, T. Milenkovic, V. Memisevic, W. Hayes, and Pržulj, "Topological network alignment uncovers biological function and phylogeny," Journal of Royal Society Interface, vol. 7, no. 50, pp.13411354, 2010.

[4] Y. Shui, and Y. R. Cho, "Alignment of PPI Networks Using Semantic Similarity for Conserved Protein Complex Prediction," IEEE Transactions on Nano Bioscience, vol. 15, no. 4, pp. 380-389, June 2016.

[5] Sourav S. Bhowmick, and Boon Siew Seah, "Clustering and Summarizing Protein Protein Interaction Networks: A Survey," IEEE Transaction on Knowledge and Data Engineering, vol. 28, no. 3, pp. 638-658, March 2016.

[6] R. Patro, and C. Kingsford, "Global network alignment using multiscale spectral signatures," Bioinformatics, vol.28, no.23, pp. 3105-3114, 2012.

[7] J. Luo, L. Yu, and Q. Dang, "Exploring the application of gene ontology semantic similarity measure for identifying protein complexes," 2014 11th International Conference on Fuzzy Systems and Knowledge Discovery (FSKD), Xiamen, 2014, pp. 502-507.

[8] A. Nagar, and H. Al-Mubaid, "A New Path Length Measure Based on GO for Gene Similarity with Evaluation using SGD Pathways," 2008 21st IEEE International Symposium on Computer-Based Medical Systems, Jyvaskyla, 2008, pp. 590-595.

[9] P. Resnik, "Using information content to evaluate semantic similarity in a taxonomy," Proc. 14th Int. Joint Conf. Artif. Intell., 1995, pp. 448$453,1995$.

[10] D.Lin, " An information-theoretic definition of similarity," Proc. 15th Int. Conf. Mach. Learn. (ICML), 1998, pp. 296-304.

[11] J. J. Jiang, and D. W. Conrath, "Semantic similarity based on corpus statistics and lexical taxonomy," Proc. 10th Int. Conf. Res. Comput. Linguistics, 1997, pp. 1-15.

[12] A. Ruepp et al., "CORUM: The comprehensive resource of mammalian protein complexes-2009," Nucleic Acids Res., vol. 36, pp. D646-D650, Jan. 2008.

[13] J. Ji, J. Lv, C. Yang, and A. Zhang, "Detecting Functional Modules Based on a Multiple-Grain Model in Large-Scale Protein-Protein Interaction Networks," IEEE/ACM Transactions on Computational Biology and Bioinformatics, vol. 13, no. 4, pp. 610-622, July-Aug. 1 2016.

[14] Y. r. Cho, W. Hwang, and A. Zhang, "Efficient Modularization of Weighted Protein Interaction Networks using k-Hop Graph Reduction," Sixth IEEE Symposium on Bio Informatics and Bio Engineering (BIBE'06), Arlington, VA, 2006, pp. 289-298. 
IEEE International Conference On Recent Trends In Electronics Information Communication Technology, May 19-20, 2017, India

[15] Kelley et al., " Pathblast: a tool for alignment of protein interaction networks," Nucleic acids research 32, no. suppl 2 (2004), pp. W83W88.

[16] Dhandra, B. V., and S. S. Patil, "An Efficient Classification of Genomes Based on Classes and Subclasses," International Journal on Computer Science and Engineering 2, no. 05 (2010): 1690-1695.

[17] Singh, Rohit, Jinbo Xu, and Bonnie Berger, "Global alignment of multiple protein interaction networks with application to functional orthology detection," Proceedings of the National Academy of Sciences 105.35 (2008): 12763-12768.

[18] Page, Lawrence, Sergey Brin, Rajeev Motwani, and Terry Winograd, "The PageRank citation ranking: Bringing order to the web," Stanford InfoLab, 1999.

[19] Tatusov et al., "The COG database: an updated version includes eukaryotes," BMC bioinformatics 4, no. 1 (2003): pp. 41.

[20] G. Kollias, S. Mohammadi, and A. Grama, "Network Similarity Decomposition (NSD): A Fast and Scalable Approach to Network Alignment," IEEE Transactions on Knowledge and Data Engineering, vol. 24, no. 12, pp. 2232-2243, Dec. 2012.

[21] Liao et al., "IsoRankN: spectral methods for global alignment of multiple protein networks," Bioinformatics (2009) 25 (12), i253-i258.

[22] Flannick et al., "Graemlin: general and robust alignment of multiple large interaction networks," Genome research 16, no. 9 (2006): 11691181.

[23] J. Hu, B. Kehr, and K. Reinert, "Netcoffee: A fast and accurate global alignment approach to identify functionally conserved proteins in multi-ple networks," Bioinformatics, vol.30, pp. 540-548, 2013.

[24] Pržulj, "Biological network comparison using graphlet degree distribution," Bioinformatics 23, no. 2 (2007): e177-e183.

[25] Mills-Tettey, G. Ayorkor, Anthony Stentz, and M. Bernardine Dias, "The Dynamic Hungarian Algorithm for the Assignment Problem with Changing Costs," Robotics Institute, Pittsburgh, PA, Tech. Rep. CMURI-TR-07-27, Jul. 2007.

[26] O. Kuchaiev, and N. Pržulj, "Integrative network alignment reveals large regions of global network similarity in yeast and human," Bioinformatics, vol.27, no.10, pp. 1390-1396, 2011.

[27] V. Memisevic, and N. Pržulj, "C-graal: Common neighbors based global graph alignment of biological networks," Integrative Biol., vol. 4, no.7, pp. 734-743, 2012.

[28] N. Malod-Dognin, N. Pržulj, "L-graal: Lagrangian graphlet based network aligner," Bioinformatics, vol.31, pp. 2182-2189, 2015.

[29] Chindelevitch, Leonid, Cheng-Yu Ma, Chung-Shou Liao, and Bonnie Berger, "Optimizing a global alignment of protein interaction networks," Bioinformatics (2013): btt486.

[30] Park, Daniel, Rohit Singh, Michael Baym, Chung-Shou Liao, and Bonnie Berger, "IsoBase: a database of functionally related proteins across PPI networks," Nucleic acids research 39, no. suppl 1 (2011): D295-D300.

[31] Sahraeian, Sayed Mohammad Ebrahim, and Byung-Jun Yoon, "A network synthesis model for generating protein interaction network families." PloS one 7, no. 8 (2012): e41474.

[32] Prasad et al., "Human protein reference database-2009 update," Nucleic acids research37, no. suppl 1 (2009): D767-D772.

[33] Stark C. et al.," BioGRID: a general repository for interaction datasets," Nucleic Acids Res 34(2006): D535-D539.

[34] Xenarios Ioannis et al., "DIP, the Database of Interacting Proteins: A Research Tool for Studying Cellular Networks of Protein Interactions," Nucleic Acids Research 30.1 (2002): 303-305.

[35] H. W. Mewes et al.," MIPS: a database for genomes and protein sequences," Nucleic Acids Research 30, no. 1 (2002): 31-34. 